\title{
Evolution of rotating binary stars
}

\author{
R. Q. Huang \\ National Astronomical Observatories/Yunnan Observatory, the Chinese Academy of Sciences, Kunmin 650011, PR China \\ e-mail: bislan@public.km.yn.cn
}

Received 2 July 2003 / Accepted 16 March 2004

\begin{abstract}
A model for the evolution of rotating binary stars is presented. The evolution of a binary system consisting of a $9 M_{\odot}$ and a $6 M_{\odot}$ star is studied in the mass transfer phase Case A. Specific attention is focused on the differences between the evolutions with and without the effects of rotation. The results indicate that the effects of rotation prolong the lifetime of the primary, and causes its evolutionary track in the HR diagram to shift towards lower luminosity. The mass and the surface helium composition of the primary become lower and the orbital period of the system becomes significantly longer during the later stage of the evolution.
\end{abstract}

Key words. stars: rotation - stars: evolution

\section{Introduction}

A series of observations indicate that rotational mixing exists in rotating stars (Herrero et al. 1992; Charbonnel 1994, 1995; Walborn 1976; Fransson et al. 1989). The evolution of rotating single stars has been studied by many investigations (Kippenhahn \& Thomas 1970; Endal \& Sofia 1976; Pinsonneaul et al. 1989; Meynet \& Maeder 1997). It is important to study the evolution of rotating binary stars. To construct a model for the evolution of binary systems with the effect of rotation, we have to take into account a series of special properties occurring in the rotating binaries: (1) The Roche potential for a rotating component consists of four terms expressing the effects of gravitation, the rotation of the component, the rotation of the system and the tide, respectively. This results in the differences between the equipotential for the rotating components and for the rotating single stars. (2) Owing to the effect of tide, the rotation of the component is solid-body rotation and synchronous with the orbital motion. (3) In the case of binary systems, we have to study not only the structure and evolution of the components, but also the evolution of the system caused by the loss of mass and angular momentum via stellar winds, the transfer of mass and angular momentum between the components and the changes in the moments of inertia of the components.

In Sect. 2, we discuss the special properties occurring in rotating binaries, and introduce a model for the binary systems with the effects of rotation. In Sect. 3, the evolution of a binary system consisting of a $9 M_{\odot}$ and a $6 M_{\odot}$ star is studied in the mass transfer phase Case A, and the differences between the results for the evolutions with and without the effects of rotation are discussed in detail.

\section{Model for rotating binary stars}

\subsection{Equipotential and the equivalent sphere}

Observations (Giuricin et al. 1984; Van Hamme \& Wilson 1990; Pan \& Tan 1998) show that most detached binary systems are synchronized systems; only a few systems are nonsynchronized. The conventional theories for the evolution of close binaries usually assume that the synchronism of the rotation with the orbital motion is always reached (Kippenhahn \& Weigert 1967; De Loore 1980; Huang \& Taam 1990; Vanbeveren 1991; De Greve 1993). The potential for a synchronous rotating component in a Roche model is given by

$$
\begin{gathered}
\Psi=\frac{G M_{1}}{r_{\mathrm{i}}}+\frac{1}{2} \omega^{2} r_{\mathrm{i}}^{2} \sin ^{2} \theta+\frac{1}{2} \omega^{2}\left(X_{\omega}-r_{\mathrm{i}} \sin \theta\right)^{2} \\
+\frac{F G M_{2}}{\sqrt{\left(A-r_{\mathrm{i}} \sin \theta\right)^{2}+r_{\mathrm{i}}^{2} \cos ^{2} \theta}}
\end{gathered}
$$

where $M_{1}$ and $M_{2}$ are the masses of the primary and the secondary, $A$ is the distance between the two components, $X_{\omega}$ is the distance between the primary and the axis through the center of mass (Fig. 1, $X_{\omega}=A M_{2} /\left(M_{1}+M_{2}\right)$ ), and $\omega$ is the orbital angular velocity of the system $\left(\omega^{2}=G\left(M_{1}+M_{2}\right) / A^{3}\right)$. The first and second terms on the right hand side of Eq. (1) correspond to the contributions of the gravitation and the rotation of the component, respectively. The third and the fourth terms correspond to the contributions of the rotation of the system and the tidal action, respectively. The basic assumption of the Roche model is that the mass of the component is concentrated in the mass center. This assumption will deviate when the configurations of the components become non-symmetric rotational ellipsoids. In such cases the value of the fourth term 


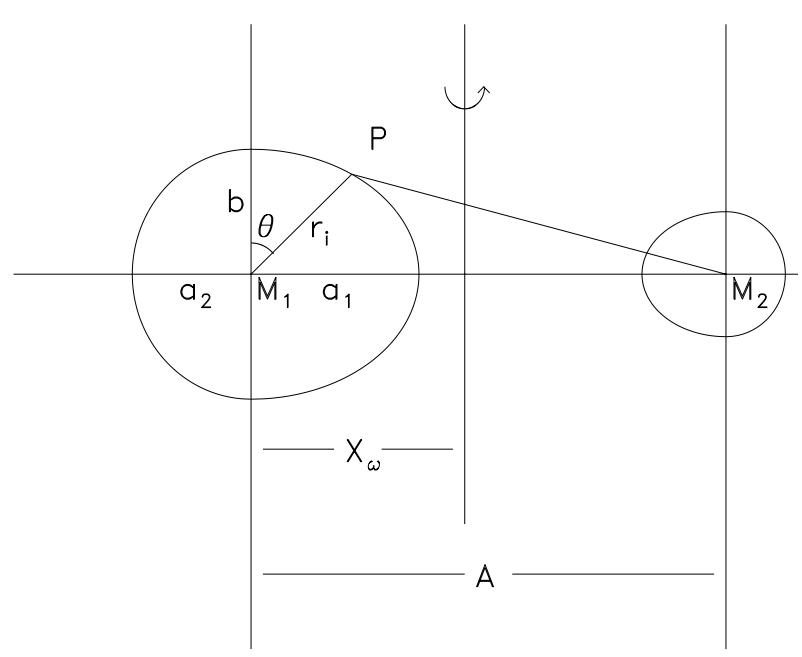

Fig. 1. Geomety of the Roche potential.

on the right hand side of Eq. (1) will decrease. Hence we introduce a factor $F$ to the fourth term in Eq. (1), and the factor $F$ may have the values between 1 and a small value during the phase of Roche lob overflow. The lowest value of this range is, however, related to the mass ratio and the distance between the two components, and is quite difficult to determine.

Introducing a quantity $q=M_{2} / M_{1}$, the dimensionless Roche potential can be obtained from (1) as:

$$
\begin{aligned}
\Psi= & \frac{G M_{1}}{A}\left[\frac{1}{\frac{r_{i}}{A}}+\frac{1}{2}(1+q)\left(\frac{r_{i}}{A}\right)^{2} \sin ^{2} \theta\right. \\
& +\frac{1}{2}(1+q)\left(\frac{q}{1+q}-\frac{r_{i} \sin \theta}{A}\right)^{2} \\
& \left.+\frac{F q}{\sqrt{\left(1-\frac{r_{i} \sin \theta}{A}\right)^{2}+\frac{r_{i}^{2} \cos ^{2} \theta}{A^{2}}}}\right] .
\end{aligned}
$$

The equipotential defined by the function $\Psi=$ const. are nonsymmetric rotational ellipsoids with two semimajor axes $a_{1}$ and $a_{2}\left(a_{1}>a_{2}\right)$ and one semiminor axis $b$ (Fig. 1). The volume $V_{\psi}$ inside the equipotential and the surface area $S_{\psi}$ of the equipotential of the primary can be expressed as

$V_{\psi}=\frac{2 \pi b}{3}\left(a_{1}^{2}+a_{2}^{2}\right)$

$S_{\psi}=\frac{4 \pi}{3}\left(a_{1}^{2}+a_{2}^{2}+b^{2}\right)$.

For any quantity, $f$, which is not constant over the equipotential surface, the mean value is defined as

$\langle f\rangle=\frac{1}{S_{\psi}} \int_{\psi=\text { const. }} f \mathrm{~d} \sigma$,

where $\mathrm{d} \sigma$ is an infinitesimal area of the surface.

We define a sphere with its volume equal to that of an equipotential ellipsoid. The radius $r_{\psi}$ of the sphere is given by

$r_{\psi}=\left(\frac{3 V_{\psi}}{4 \pi}\right)^{1 / 3}$.

This sphere is called the volume equivalent sphere, and its radius's called the equivalent radius of the equipotential ellipsoid.

\subsection{The interior stellar structure equations}

Due to the effect of tide, the rotation of the component is synchronous with the orbital motion of the system. Such synchronous rotation exists also in the interior of the component. Thus, the rotation of the component is solid-body rotation and conservative. Kippenhahn \& Thomas (1970) introduced a method to simplify the two-dimensional model with conservative rotation to a one dimensional model, and gave the structure equations as follows:

$$
\begin{aligned}
\frac{\mathrm{d} r_{\Psi}}{\mathrm{d} M_{\Psi}} & =\frac{1}{4 \pi r_{\Psi}^{2} \rho}, \\
\frac{\mathrm{d} P}{\mathrm{~d} M_{\psi}} & =-\frac{G M_{\Psi}}{4 \pi r_{\Psi}^{2}} f_{\mathrm{P}},
\end{aligned}
$$

$\frac{\mathrm{d} L}{\mathrm{~d} M_{\Psi}}=\varepsilon_{N}-\varepsilon_{\nu}+\varepsilon_{g}$,

$\frac{\mathrm{d} \ln T}{\mathrm{~d} \ln P}=\left\{\begin{array}{c}\nabla_{\mathrm{R}} f_{T} / f_{P} \\ \nabla_{\mathrm{con}}\end{array}\right.$

where

$f_{P}=\frac{4 \pi r_{\Psi}^{4}}{G M_{\Psi} S_{\Psi}} \frac{1}{\left\langle g_{\mathrm{eff}}^{-1}\right\rangle}$,

$f_{T}=\left(\frac{4 \pi r_{\Psi}^{2}}{S_{\Psi}}\right)^{2} \frac{1}{\left\langle g_{\mathrm{eff}}\right\rangle\left\langle g_{\mathrm{eff}}^{-1}\right\rangle}$,

$\nabla_{\mathrm{R}}=\frac{3 \kappa L P}{4 a c G M_{\Psi} T^{4}}$,

here, $\left\langle g_{\mathrm{eff}}\right\rangle,\left\langle g_{\mathrm{eff}}^{-1}\right\rangle$ are the mean values of the effective gravity and its inverse over the equipotential surface, $\nabla_{R}$ the radiative temperature gradient.

\subsection{Calculations of the quantities $f_{P}$ and $f_{T}$}

According to Eqs. (11) and (12), the key to get the values of $f_{P}$ and $f_{T}$ is to calculate the mean values $\left\langle g_{\text {eff }}\right\rangle$ and $\left\langle g_{\text {eff }}^{-1}\right\rangle$. In practical calculations, the two-dimensional model for the rotating component is simplified to a one-dimensional model. Thus, the stratification of the component with non-spherical equipotential is replaced by the stratification with equivalent spheres. The calculation of the mean values of $\left\langle g_{\text {eff }}\right\rangle$ and $\left\langle g_{\text {eff }}^{-1}\right\rangle$ over the equipotential surface is divided, therefore, into two steps: the first step is to get the equipotential surface by giving the corresponding equivalent sphere, the second step is to calculate the mean values of $\left\langle g_{\mathrm{eff}}\right\rangle$ and $\left\langle g_{\mathrm{eff}}^{-1}\right\rangle$ over the equipotential surface.

\subsubsection{Replacement of equipotential by the equivalent sphere}

Assuming $m_{\Psi}$ is the mass in the equivalent sphere of radius $r_{\Psi}$, and the quantity $q_{\Psi}=\frac{m_{\Psi}}{M_{1}}$, the functions for the semi-axis $a_{1}, a_{2}$ 
and $b$ to the $r_{\psi}$ of the equipotential can be obtained from Eqs. (1), (3) and (4) as

$$
\frac{2 \pi b}{3}\left(a_{1}^{2}+a_{2}^{2}\right)=\frac{4 \pi r_{\Psi}^{3}}{3}
$$

$$
\begin{aligned}
\frac{G M_{1}}{A}\left[\frac{q_{\psi}}{a_{1 / A}}+\right. & \frac{1}{2}(1+q)\left(\frac{a_{1}}{A}\right)^{2} \\
& \left.+\frac{1}{2}(1+q)\left(\frac{q}{1+q}-\frac{a_{1}}{A}\right)^{2}+\frac{F q}{1-\frac{a_{1}}{A}}\right] \\
& =\frac{G M_{1}}{A}\left[\frac{q \Psi}{b / A}+\frac{1}{2} \frac{q^{2}}{1+q}+\frac{F q}{\sqrt{1+\left(\frac{b}{A}\right)^{2}}}\right],
\end{aligned}
$$

$$
\begin{aligned}
\frac{G M_{1}}{A}\left[\frac{q_{\psi}}{a_{2 / A}}+\right. & \frac{1}{2}(1+q)\left(\frac{a_{2}}{A}\right)^{2} \\
& \left.+\frac{1}{2}(1+q)\left(\frac{q}{1+q}+\frac{a_{2}}{A}\right)^{2}+\frac{F q}{1+\frac{a_{2}}{A}}\right] \\
& =\frac{G M_{1}}{A}\left[\frac{q \Psi}{b / A}+\frac{1}{2} \frac{q^{2}}{1+q}+\frac{F q}{\sqrt{1+\left(\frac{b}{A}\right)^{2}}}\right],
\end{aligned}
$$

where $q=M_{2} / M_{1}$. The semi-axis $a_{1}, a_{2}$ and $b$ can be obtained by solving Eqs. (14)-(16) numerically.

\subsubsection{Calculations of $\left\langle g_{\text {eff }}\right\rangle$ and $\left\langle g_{\text {eff }}^{-1}\right\rangle$}

It is difficult to calculate the mean value of a quantity $f$ over the equipotential surface directly, because the equipotential is a non-symmetric rotational ellipsoid with two semimajor axes $a_{1}$ and $a_{2}\left(a_{1}>a_{2}\right)$. We imagine that the non-symmetric rotational ellipsoid is composed of two parts. One part is a half of a symmetric rotational ellipsoid with semimajor axis $a_{1}$ and semiminor axis $b$ (The dot-dashed curve in Fig. 2). The other part is also a half of a symmetric rotational ellipsoid with semimajor axis $a_{2}$ and semiminor axis $b$ (The dotted curve in Fig. 2). The calculation of the mean value of a quantity $f$ over the equipotential surface can be replaced, therefore, by calculations of the mean values over the two symmetric rotational ellipsoids.

Assuming $m_{\Psi}$ is the mass in the equipotential, and the quantity $q_{\Psi}=\frac{m_{\Psi}}{M_{1}}$, the Roche potential of the two symmetric rotational ellipsoids can be written from Eq. (2) as

$$
\begin{aligned}
\Psi_{1}= & \frac{G M_{1}}{A}\left[\frac{q_{\psi}}{\frac{r_{1 i}}{A}}+\frac{1}{2}(1+q)\left(\frac{r_{1 i}}{A}\right)^{2} \sin ^{2} \theta\right. \\
& +\frac{1}{2}(1+q)\left(\frac{q}{1+q}-\frac{r_{1 i} \sin \theta}{A}\right)^{2} \\
& \left.+\frac{F q}{\sqrt{\left(1-\frac{r_{1 i} \sin \theta}{A}\right)^{2}+\frac{r_{1 i}^{2} \cos ^{2} \theta}{A^{2}}}}\right],
\end{aligned}
$$

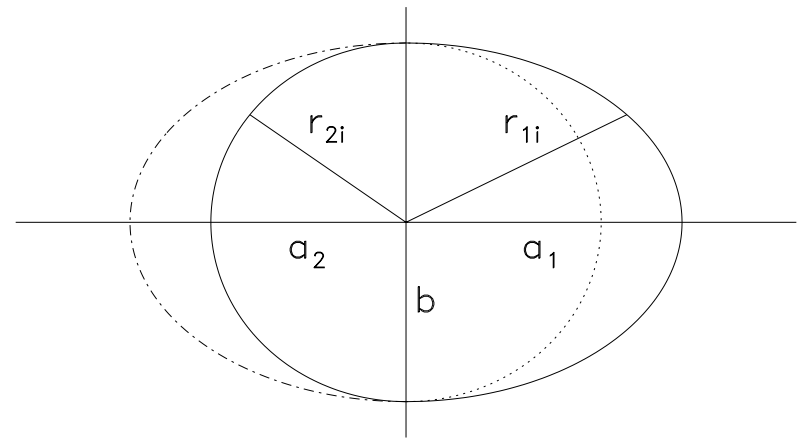

Fig. 2. Geometry of the equipotential. The solid curve is a nonsymmetric rotational ellipsoid with two semimajor axes $a_{1}$ and $a_{2}$ and one semiminor axis $b$. The dot-dashed curve is a half of a symmetric rotational ellipsoid with semimajor axis $a_{1}$ and semiminor axis $b$. The dotted curve is also a half of a symmetric rotational ellipsoid with semimajor axis $a_{2}$ and semiminor axis $b$.

$$
\begin{aligned}
\Psi_{2}= & \frac{G M_{1}}{A}\left[\frac{q_{\psi}}{\frac{r_{2 i}}{A}}+\frac{1}{2}(1+q)\left(\frac{r_{2 i}}{A}\right)^{2} \sin ^{2} \theta\right. \\
& +\frac{1}{2}(1+q)\left(\frac{q}{1+q}+\frac{r_{2 i} \sin \theta}{A}\right)^{2} \\
& \left.+\frac{F q}{\sqrt{\left(1+\frac{r_{2 i} \sin \theta}{A}\right)^{2}+\frac{r_{12}^{2} \cos ^{2} \theta}{A^{2}}}}\right]
\end{aligned}
$$

where $r_{1 i}$ and $r_{2 i}$ are the distances between the center of the component and the surfaces of the two symmetric ellipsoids (see Fig. 2). The relationships of $r_{1 i}$ and $r_{2 i}$ to the semi axis of the two symmetric ellipsoids are given by

$$
\begin{aligned}
& r_{1 i}^{2}=\frac{a_{1}^{2} b^{2}}{a_{1}^{2} \cos ^{2} \theta+b^{2} \sin ^{2} \theta}, \\
& r_{2 i}^{2}=\frac{a_{2}^{2} b^{2}}{a_{2}^{2} \cos ^{2} \theta+b^{2} \sin ^{2} \theta} .
\end{aligned}
$$

The effective gravitational accelerations on the surfaces of the two symmetric ellipsoids are given by

$g_{\mathrm{eff} 1}=\sqrt{\left(\frac{\partial \Psi_{1}}{\partial r_{1 i}}\right)^{2}+\left(\frac{1}{r_{1 i}} \frac{\partial \Psi_{1}}{\partial \theta}\right)^{2}}$

$g_{\mathrm{eff} 2}=\sqrt{\left(\frac{\partial \Psi_{2}}{\partial r_{2 i}}\right)^{2}+\left(\frac{1}{r_{2 i}} \frac{\partial \Psi_{2}}{\partial \theta}\right)^{2}}$

The mean values of $g_{\text {eff1 }}$ and $g_{\text {eff2 }}$ over the surfaces of the two symmetric ellipsoids are given by

$$
\begin{aligned}
& \left\langle g_{\text {eff } 1}\right\rangle=\frac{1}{S_{\Psi 1}} \int_{0}^{\pi / 2} g_{\text {eff } 1} 2 \pi r_{1 i}^{2} \sin \theta \mathrm{d} \theta, \\
& \left\langle g_{\text {eff } 2}\right\rangle=\frac{1}{S_{\Psi 2}} \int_{0}^{\pi / 2} g_{\text {eff } 2} 2 \pi r_{2 i}^{2} \sin \theta \mathrm{d} \theta,
\end{aligned}
$$


where

$S_{\Psi 1}=\frac{4 \pi}{3}\left(2 a_{1}^{2}+b^{2}\right)$,

$S_{\Psi 2}=\frac{4 \pi}{3}\left(2 a_{2}^{2}+b^{2}\right)$.

The mean value of the effective gravitational acceleration over the equipotential surface is obtained by

$\left\langle g_{\mathrm{eff}}\right\rangle=\frac{1}{S_{\Psi 1}+S_{\Psi 2}}\left(S_{\Psi 1}\left\langle g_{\mathrm{eff} 1}\right\rangle+S_{\Psi 2}\left\langle g_{\mathrm{eff} 2}\right\rangle\right)$.

According to Eqs. (11) and (12), the values of $f_{P}$ and $f_{T}$ can be obtained when the mean values $\left\langle g_{\text {eff }}\right\rangle$ and $\left\langle g_{\text {eff }}^{-1}\right\rangle$ are known.

\subsection{Rotational mixing}

The effects of meridian circulation and shear turbulence can drive transport of the chemical elements and the angular momentum in rotating stars. For the components with solid-body rotation there exists no differential rotation that can drive shear turbulence. Hence the rotational mixing in a rotating component can be driven only by the effect of meridian circulation. Owing to the effect of tidal motion, the calculation of the meridian circulation for the rotating components are much more complicated than that for the single rotating stars. Hence we use a simplified method to treat the transport of the chemical elements as a diffusion-advection process. Due to the fact that the transport of the chemical compositions is caused by the effect of rotation, and the effect of rotation of a mass layer can be expressed by the ratio of the mean effective gravity $\left\langle g_{\mathrm{eff}}\right\rangle_{i}$ to the gravity $g_{i}$ of this layer, so the change of the composition of the element $\alpha$ via a diffusion-advection process can be expressed as:

$$
\begin{aligned}
\left(\frac{\partial X_{\alpha}}{\partial t}\right)_{r}= & \left(\frac{\partial}{\partial r}\right)_{t}\left[-D_{\mathrm{adv}} \frac{\left\langle g_{\mathrm{eff}}\right\rangle_{i}}{g_{i}}-D_{\mathrm{dif}}\left(\frac{\partial X_{\alpha}}{\partial r}\right)_{t}\right] \\
& +\left(\frac{\mathrm{d} X_{\alpha}}{\mathrm{d} t}\right)_{\mathrm{nuc}},
\end{aligned}
$$

where $D_{\text {adv }}$ and $D_{\text {dif }}$ are the coefficients $\left(D_{\text {adv }}=\frac{K_{1}}{\tau_{\text {adv }}}, D_{\text {dif }}=\right.$ $\left.\frac{K_{2}}{\tau_{\text {th }}}\right), K_{1}, K_{2}$ are the dimensionless parameters. The thermal and the advection time-scales can be given as (see Maeder \& Meynet 2000; Huang \& Yu 1998): $\tau_{\text {th }}=\frac{1}{2} q \frac{G M^{2}}{R L}, q=1.5$ and $\tau_{\mathrm{adv}}=\tau_{\mathrm{th}} \frac{G M}{R^{3} \omega^{2}}$. The last term of Eq. (28) is the change of the composition due to the nuclear reaction.

At the inner and outer boundary reflecting conditions are used:

$\left.\left(\frac{\partial X_{\alpha}}{\partial r}\right)_{t}\right|_{r=0}=0=\left.\left(\frac{\partial X_{\alpha}}{\partial r}\right)_{t}\right|_{r=R}$.

\subsection{Mass loss}

Among the numerous observational and theoretical investigations on the mass loss rate via stellar winds we adopt the empirical mass loss rate law proposed by Nieuwenhuijzen \& Jager (1990):

$$
\begin{aligned}
\log (-\dot{M})= & -1.61 \log T_{\text {eff }}+1.64 \log \left(L / L_{\odot}\right) \\
& +0.161 \log \left(M / M_{\odot}\right)-7.93 .
\end{aligned}
$$

The rate of mass loss from a rotating component star in a binary system may be different from that from a rotating single star of the same spectral type and luminosity class due to the effects of tide, and due to the centrifugal forces caused by the rotation of the system. From Friend \& Castor (1982), the mass loss rate is related to the gravitational potential. Therefore, the rate of mass loss for a component star is enhanced by a factor $f$, which is given by Huang \& Taam (1990) as

$f=\frac{g}{\left\langle g_{\mathrm{eff}}\right\rangle}$,

where $g$ is the gravity at the surface of the volume equivalent sphere, $\left\langle g_{\text {eff }}\right\rangle$ the mean effective gravity of this layer.

\subsection{Condition for mass exchange}

In the case without the effect of rotation, the components are spherically symmetric, and the condition for the mass exchange via Roche lobe overflow is when the radius of the component is equal to that of the Roche lobe. However, in the case with the effect of rotation, the components are non-symmetric rotational ellipsoids with the two semimajor axes $a_{1}$ and $a_{2}\left(a_{1}>a_{2}\right)$. The condition for the mass exchange through Roche lobe flow over is changed to that when the longer semimajor axis $a_{1}$ of the component is equal to the critical radius of the Roche lobe.

\subsection{Changes in the angular velocity of the system and the orbital separation between the two components}

The angular velocity of the system and the orbital separation between the two components are variables due to the many physical processes occurring in the binary system during evolution, such as the loss of mass and angular momentum via stellar winds, the exchange of mass via Roche lobe overflow, the exchange of angular momentum between the rotation of the components and the orbital motion of the system caused by the effect of tide, and the change in the moments of inertia of the components. According to Huang \& Taam (1990), the changes in the angular velocity of the system and the orbital separation between the two components can be obtained by

$$
\begin{aligned}
\frac{\Delta \omega}{\omega}= & \frac{1}{2} \frac{\Delta M_{1}+\Delta M_{2}}{M_{1}+M_{2}}-\frac{3}{2} \frac{\Delta A}{A}, \\
\frac{\Delta A}{A}= & \frac{1}{\frac{1}{2}-2 \beta}\left[\frac{\Delta J}{J}-\frac{\Delta M_{1}}{M_{1}}(1-\beta)-\frac{\Delta M_{2}}{M_{2}}(1-\beta)\right. \\
& \left.-\frac{\Delta M_{1}+\Delta M_{2}}{M_{1}+M_{2}}\left(\beta-\frac{1}{2}\right)-\frac{\Delta I_{1}+\Delta I_{2}}{I_{1}+I_{2}} \beta\right],
\end{aligned}
$$


where $\Delta M_{1}, \Delta M_{2}$ are the changes in masses of the primary and the secondary, and are given by

$$
\begin{aligned}
& \Delta M_{1}=\Delta M_{1 c}+\Delta M_{1 w}, \\
& \Delta M_{2}=\Delta M_{2 c}+\Delta M_{2 w} .
\end{aligned}
$$

The first and second terms on the right hand side of Eqs. (34) and (35) correspond the changes of mass via Roche lobe flow over and the mass loss via stellar winds, respectively.

In Eqs. (30) and (31), $J$ is the total angular momentum of the system, and can be written as

$J=J_{0}+\left(I_{1}+I_{2}\right) \omega$

where

$J_{0}=\frac{G^{1 / 2} A^{1 / 2} M_{1} M_{2}}{\left(M_{1}+M_{2}\right)^{1 / 2}}$,

here $J_{0}$ is the orbital angular momentum of the system, $I_{1}, I_{2}$ are the moments of inertia of the primary and of the secondary, $\left(I_{1}+I_{2}\right) \omega$ is the rotational angular momentum, and $\beta=\left(I_{1}+I_{2}\right) \omega / J$ is the ratio of rotational angular momentum to the total angular momentum of the system.

The loss of angular momentum carried away by stellar winds are given by

$$
\begin{aligned}
\Delta J= & \left(\frac{2}{3} R_{1}^{2}+X_{\omega}^{2}\right) \Delta M_{1 w} \omega \\
& +\left(\frac{2}{3} R_{2}^{2}+\left(a-X_{\omega}\right)^{2}\right) \Delta M_{2 w} \omega,
\end{aligned}
$$

where $R_{1}$ and $R_{2}$ are the radius of the primary and the secondary, $X_{\omega}$ is the distance between the primary and the axis through the center of mass (see Fig. 1).

\section{Evolutionary computations and results}

The evolution of a binary system consisting of a $9 M_{\odot}$ and a $6 M_{\odot}$ star is studied. Both components of the binary system are treated simultaneously including mass transfer and the effect of rotation. Two evolutionary sequences corresponding to the cases with and without the effect of rotation are calculated. The sequence denoted as STD corresponds to the evolution without the effect of rotation, while the sequence denoted as ROT corresponds to that with the effect of rotation. The initial chemical composition of $X=0.70, Z=0.02$ is adopted for the components of the two sequences. The parameters $K_{1}$ and $K_{2}$ in Eq. (28) have the values of $10^{-4}$ and $10^{-6}$. The initial orbital separation between the two components in the two sequences is chosen to be $20.771 R_{\odot}$ so that the mass transfer via Roche lobe occurs in Case A (during the central hydrogen-burning phase of the primary). The effects of mass loss due to stellar winds and convective overshooting are considered for both sequences.

The evolutionary tracks of the primary in the HR diagram for the two sequences are illustrated in Fig. 3. The solid curve corresponds to the track of the STD sequence, and the dotted curve corresponds to that of the ROT sequence. Points $a, b$, $\mathrm{c}, \mathrm{d}$ and $\mathrm{e}$ on the tracks denote the zero age main sequence, the beginning of the mass transfer phase, the end of the central

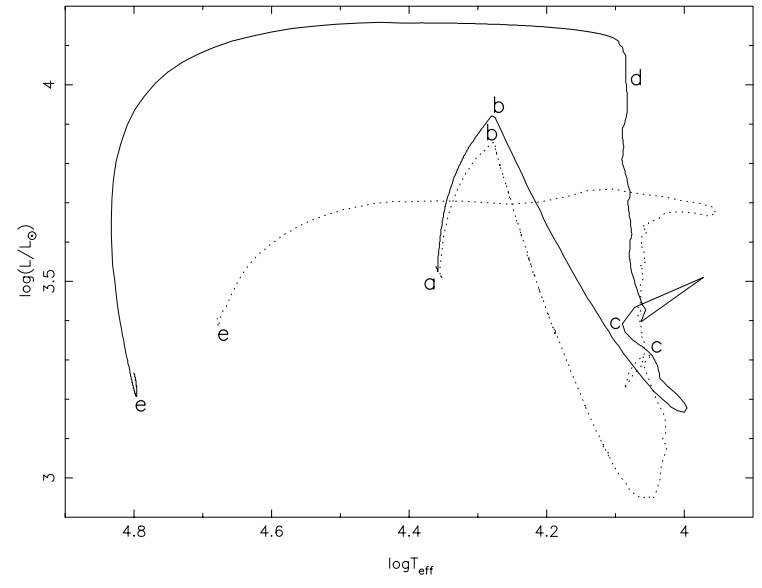

Fig. 3. The evolutionary tracks of the primary in HR diagram for the two sequences. The solid curve corresponds to the track of the STD sequence, and the dotted curve corresponds to that of the ROT sequence. Points $\mathrm{a}, \mathrm{b}, \mathrm{c}, \mathrm{d}$ and e on the tracks denote the zero age main sequence, the beginning of the mass transfer phase, the end of the central hydrogen-burning phase, the beginning of the central helium-burning phase and the end of the calculations, respectively.

hydrogen-burning phase, the beginning of the central heliumburning phase and the end of the calculations, respectively. From the difference between the solid and dotted curves in Fig. 3 one finds that the effect of rotation causes the evolutionary track of the primary to shift towards lower luminosity.

In Table 1 we list the ages, orbital periods, masses of the primary and secondary, luminosities, effective temperatures, the central hydrogen and helium compositions, the compositions of hydrogen and helium on the surface and the equatorial velocity of the primary at the critical evolutionary points of the sequences. From the parameters at point $\mathrm{b}$ in Table 1 one finds that the mass transfer phase begins at $t=2.8895 \times 10^{7} \mathrm{yr}$ for the STD sequence and at $2.8200 \times 10^{7} \mathrm{yr}$ for the ROT sequence. Thus, the time of the beginning of mass transfer phase for the ROT sequence advances by $\sim 2.4 \%$. This can be understood by the fact that the primary is a non-symmetric rotational ellipsoid due to the effect of rotation, the longer semimajor axis is longer than the equivalent radius, and the criterion for the onset of mass transfer is that the longer semimajor axis equals to the radius of the Roche lobe. Due to the difference between the beginning times of mass transfer, the central hydrogen composition of the primary at point $b$ has a value of $X(c)=0.0567$ for the STD sequence instead of 0.1305 for the ROT sequence.

In Table 1 we find that the STD sequence reaches point $\mathrm{c}$ at $t=3.1239 \times 10^{7} \mathrm{yr}$, while the ROT sequence reaches point $\mathrm{c}$ at $t=3.5303 \times 10^{7} \mathrm{yr}$. Thus, the ROT sequence is later by about $10.7 \%$ for reaching point c. Furthermore, we find in Table 1 that the central helium-burning begins at the point $\mathrm{d}$ at $t=3.1527 \times 10^{7} \mathrm{yr}$ for the STD sequence, and there is still no central helium-burning occurring at the later poind e with the time $t=3.6827 \times 10^{7} \mathrm{yr}$ for the ROT sequence. This means that the effect of rotation causes the primary to evolve slower.

In addition, one finds in Table 1 that the surface helium composition of the primary has a value of $Y=0.3307$ at point $\mathrm{c}$, and $Y=0.9351$ at point e for the STD sequence, 
Table 1. The parameters at different evolutionary points a, b, c, d and e of the STD and ROT sequences.

\begin{tabular}{|c|c|c|c|c|c|c|c|c|c|c|c|}
\hline Sequence & $t\left(10^{7} \mathrm{yr}\right)$ & $P(\mathrm{~d})$ & $M_{1}\left(M_{\odot}\right)$ & $M_{2}\left(M_{\odot}\right)$ & $\log L / L_{\odot}$ & $\log T_{\text {eff }}$ & $X_{1}(c)$ & $Y_{1}(c)$ & $X_{1}$ & $Y_{1}$ & $V_{\text {rot }}\left(\mathrm{km} \mathrm{s}^{1}\right)$ \\
\hline \multicolumn{12}{|l|}{$\mathrm{a}$} \\
\hline STD & 0.0000 & 2.775 & 9.000 & 6.000 & 3.538 & 4.361 & 0.700 & 0.2800 & 0.7000 & 0.2800 & \\
\hline ROT & 0.0000 & 2.773 & 9.000 & 6.000 & 3.512 & 4.353 & 0.700 & 0.2800 & 0.7000 & 0.2800 & 68.48 \\
\hline \multicolumn{12}{|l|}{$\mathrm{b}$} \\
\hline STD & 2.8859 & 2.727 & 8.935 & 5.995 & 3.927 & 4.278 & 0.0567 & 0.9238 & 0.7000 & 0.2800 & \\
\hline ROT & 2.8200 & 2.738 & 8.942 & 5.996 & 3.853 & 4.277 & 0.1305 & 0.8495 & 0.6999 & 0.2800 & 145.92 \\
\hline \multicolumn{12}{|l|}{$\mathrm{c}$} \\
\hline STD & 3.1239 & 9.116 & 3.030 & 11.885 & 3.434 & 4.072 & 0.0000 & 0.9800 & 0.6493 & 0.3307 & \\
\hline ROT & 3.5338 & 9.136 & 3.045 & 11.840 & 3.305 & 4.063 & 0.0000 & 0.9800 & 0.6662 & 0.3138 & 62.61 \\
\hline \multicolumn{12}{|l|}{$\mathrm{d}$} \\
\hline STD & 3.1527 & 31.726 & 1.803 & 13.094 & 4.060 & 4.085 & 0.0000 & 0.9799 & 0.1139 & 0.8661 & \\
\hline \multicolumn{12}{|l|}{$\mathrm{e}$} \\
\hline STD & 3.3969 & 34.051 & 1.694 & 13.113 & 3.267 & 4.800 & 0.0000 & 0.4006 & 0.0449 & 0.9351 & \\
\hline ROT & 3.6827 & 52.508 & 1.461 & 13.366 & 3.406 & 4.679 & 0.0000 & 0.9800 & 0.1268 & 0.8532 & 0.71 \\
\hline
\end{tabular}

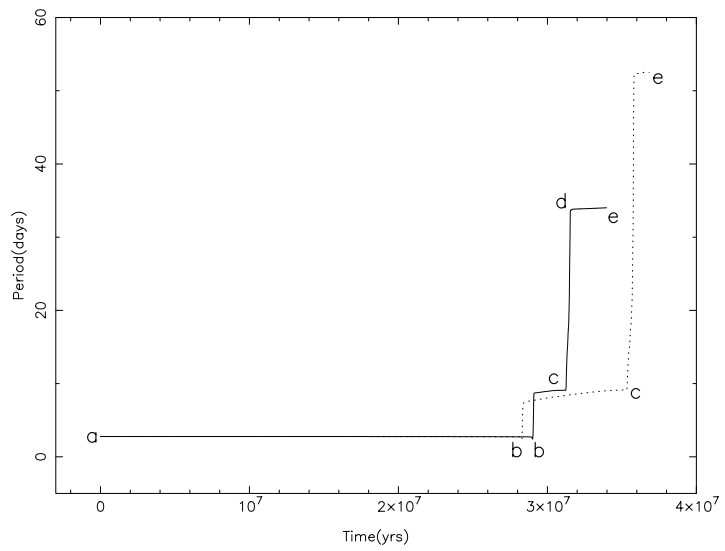

Fig. 4. The time dependent variation in the orbital period of the system. The solid and dotted curves and the points a, b, c, d, and e have the same meanings as in Fig. 3.

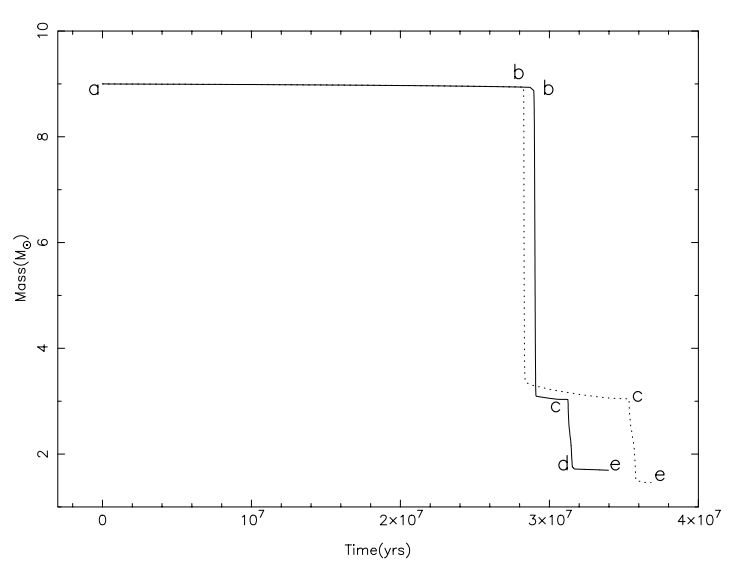

Fig. 5. The time dependent variation in the mass of the primary. The solid and dotted curves and the points a, b, c, d, and e have the same meanings as in Fig. 3.

while $Y=0.3138$ at point $\mathrm{c}$ and $Y=0.8532$ at point $\mathrm{e}$ for the ROT sequence. Thus, the effect of rotation makes the surface helium composition of the primary smaller during the later stage of the evolution.

Figures 4 and 5 illustrate the time dependent variation in the orbital period of the system and the mass of the primary. The solid and the dotted curves in Figs. 4 and 5 correspond to the STD and ROT sequence, respectively. Comparisons of the orbital periods and the masses of the primary at point $d$ between the two sequences show that the effect of rotation causes the orbital period of the system to increases by about $54 \%$, and the mass of the primary to decrease by about $14 \%$.

Acknowledgements. This work is supported by the Chinese National Science Foundation No. 10073020P.

\section{References}

Charbonnel, C. 1994, A\&A, 282, 811

Charbonnel, C. 1995, ApJ, 453, L41

Chiosi, C., \& Maeder, A. 1986, ARA\&A, 24, 329

Endal, A. S., \& Sofia, S. 1976, ApJ, 210, 184

Fransson, C., Cassatella, A., Gilmozzi, R., et al. 1989, ApJ, 336, 429

Friend, D. B., \& Castor, J. I. 1982, ApJ, 261, 293

Giuricin, G., Mardirossian, F., \& Mezzeti, M. 1984, A\&A, 131, 152

De Greve, J. P. 1993, A\&A, 97, 527

Van Hamme, W., \& Wilson, R. E. 1990, AJ, 100, 1981

Herrero, A., Kudritzki, R. P., Vilcheez, J. M., et al. 1992, A\&A, 261, 209

Huang, R. Q., \& Taam, R. E. 1990, A\&A, 236, 107

Huang, R. Q., \& Yu, K. N. 1998, Stellar Astrophysics (SpringerVerlag), 232

Kippenhahn, R., \& Weigert, A. 1967, ApJ, 65, 251

Kippenhahn, R., \& Thomas, H. C. 1970, In Stellar Rotation, ed. A. Slettebak (Holland: D. Reidel Publ. Co. Dordrecht), 20

De Loore, C. 1980, Space Sci. Rev., 26, 113

Maeder, A., \& Meynet, G. 2000, ARA\&A, 38, 143

Meynet, G., \& Maeder, A. 1997, A\&A, 321, 465

Nieuwenhuijzen, H., \& de Jager, C. 1990, A\&A, 231, 134

Pan, K., \& Tan, H. 1998, A\&A, 335, 179

Pinsonneault, M. H., Kawaler, S. D., Sofia, S., \& Demarqure, P. 1989, ApJS, 338, 424

Vanbeveren, D. 1991, Space Sci. Rev., 56, 249

Walborn, N. R. 1976, ApJ, 205, 419 